\title{
AUTOMATIC 1D INVERSION OF ELECTRICAL WELL LOGS USING THE L1-NORM AND FIXED LAYER-BOUNDARIES
}

\author{
Max Halkjar \\ Kemp \& Lauritzen Vand \& Miljø A/S, Michael Drewsensvej 23, DK-8270 Højbjerg, Phone \\ +4586291266 - Fax +4586299372 - e-mail mhalkjaer@hotmail.com
}

\section{INTRODUCTION}

For electrical well logs, it is well known that the measured apparent resistivity differs from the true formation resistivity in a region around boundaries between formations with different electrical resistivity. The apparent resistivity varies with electrode configuration and with resistivity contrast. In this paper, it is shown that inversion of the electrical log leads to a substantial increased resolution of the lithologic sequence. The inversion is performed with a simple one dimensional (1-D) L1-norm inversion routine which can accommodate for a very high number of layers with fixed layer boundaries.

\section{METHOD}

The inversion model is a multi layer model (MLM) defined by thin horizontal layers with fixed layer boundaries. In this way, the only free parametre is the layer resistivities. The inversion is stabilized with elastic bonds between the layer resistivities. Test has shown that the stabilizing resistivity bands make the inversion converge rapidly, typical after only 4 iterations, and the initial model can be chosen to be a homogenous fullspace. This makes the inversion fully automatic and very objective.

The minimization is done in the L1-norm (Madsen and Nielsen, 1993). A comparison of the L1-norm and the L2-norm (least squares method) has shown, that the L1-norm results in better defined resistivity shifts. Futhermore, unwanted oscillations in the obtained model are supressed.

On the basis of the Frechet kernel for a given electrode configuration, the influenced area in the neighbourhood of the electrodes can be estimated. With that in mind, the inversion can be done piece by piece with a certain overlap. This is done to reduce the number of input data and model parameters. The model results are then combined as the model layers close to the boundaries are removed.

The choice of layer thickness and stabilizing resistivity bands determine the resolution. The optimum is determind by a synthetic test. The inversion test were mainly based on analytically calculated two and three layer models perturbated with 5 percent Gaussian 
distributed noise. A factor of 10 was selected for the resistivity contrast.

It was found that for a Wenner log based on 20 measurements per electrode spacing $(\mathrm{a} / 20)$, a layer thickness of 0.25 times the electrode distance and a resistivity band of 10 percent is preferable. These parameters cause a resolution as high as half the electrode spacing. For a Normal log configuration, similar synthetic tests were done. It was found that the layer thickness should be 0.25 times the electrode distance as for the Wenner arrays but the resistivity band must be tighter $(2.5 \%)$.

The inversion has been done with $1 \mathrm{D}$ electrical and electromagnetic inversion program SELMA (Christensen and Auken, 1992) provided with a shell, with data prefiltering and input - output facilities.

\section{A THEORETICAL DATASET}

Figure 1a shows a 5 layer model. An apparent resistivity Wenner electrode configuration has been used. As shown, it is impossible to determine the true resistivity from the apparent resistivity, and therefore the geological strata are not resolved satisfactorily.

Figure $1 \mathrm{~b}$ shows the same data set perturbated with $5 \%$ noise (dots) and inverted with the MLM-method (layer thicknes $=0.25$ times the electrode spacing and resistivity band $=10 \%$ ). As it can be seen, the model comes close to the true resistivity, i.e. the four geological layer boundaries are well defined and the difference between the geolgical model and the inversion model is small.
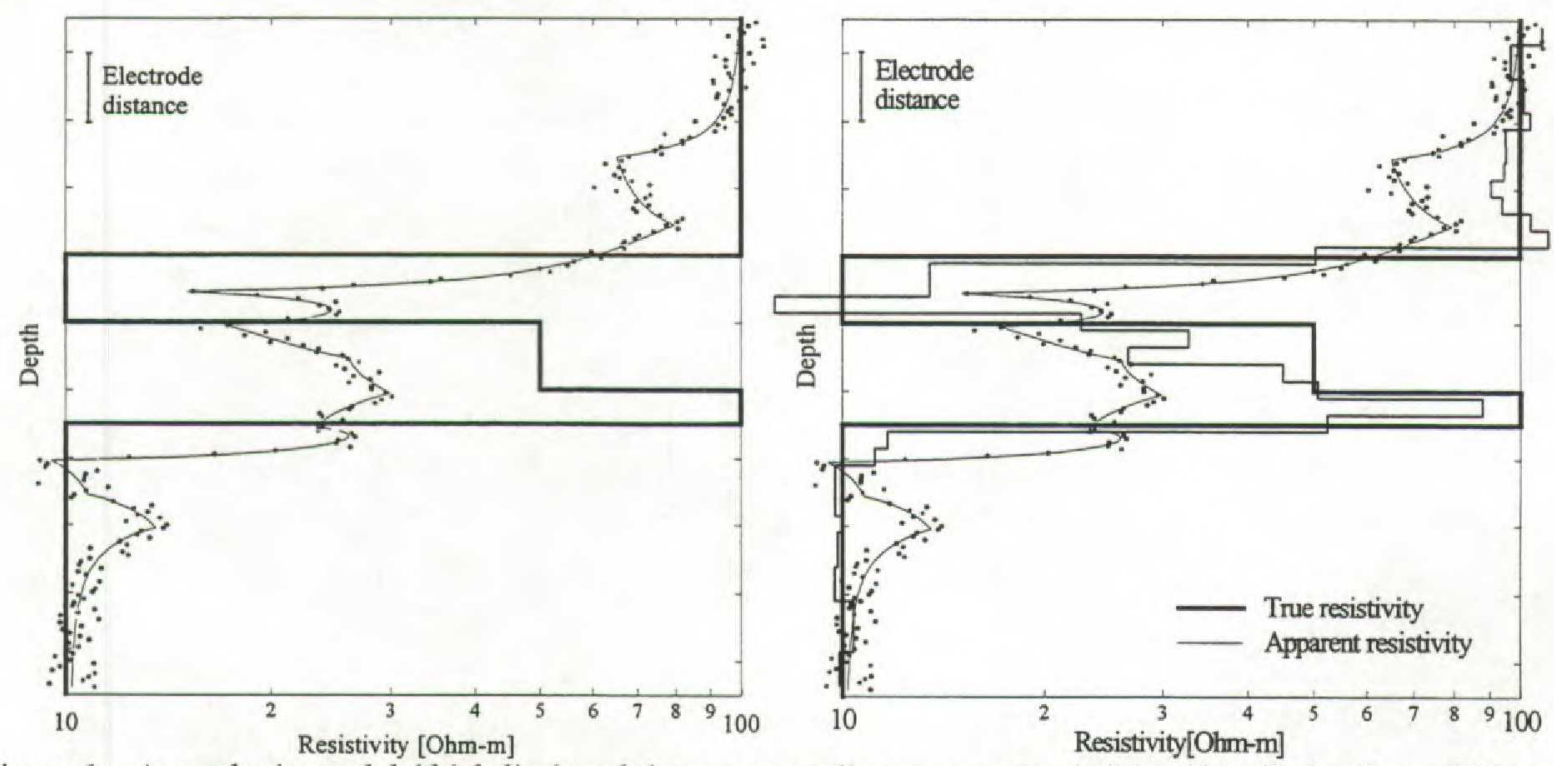

Figure 1a. A synthetic model (thick line) and the corresponding apparent resistivity (thin line), when a Wenner electrode configuration with an electrode spacing, a, is used. The dots are the apparent resistivity pertubated with $5 \%$ Gaussian distributed noise.

Figure $1 \mathrm{~b}$. The same as figure 1 supplemented with the inversion model result.

\section{FIELD EXAMPLE}

Figure 2 shows a section from a Wenner log acquired with the ellog drilling method 


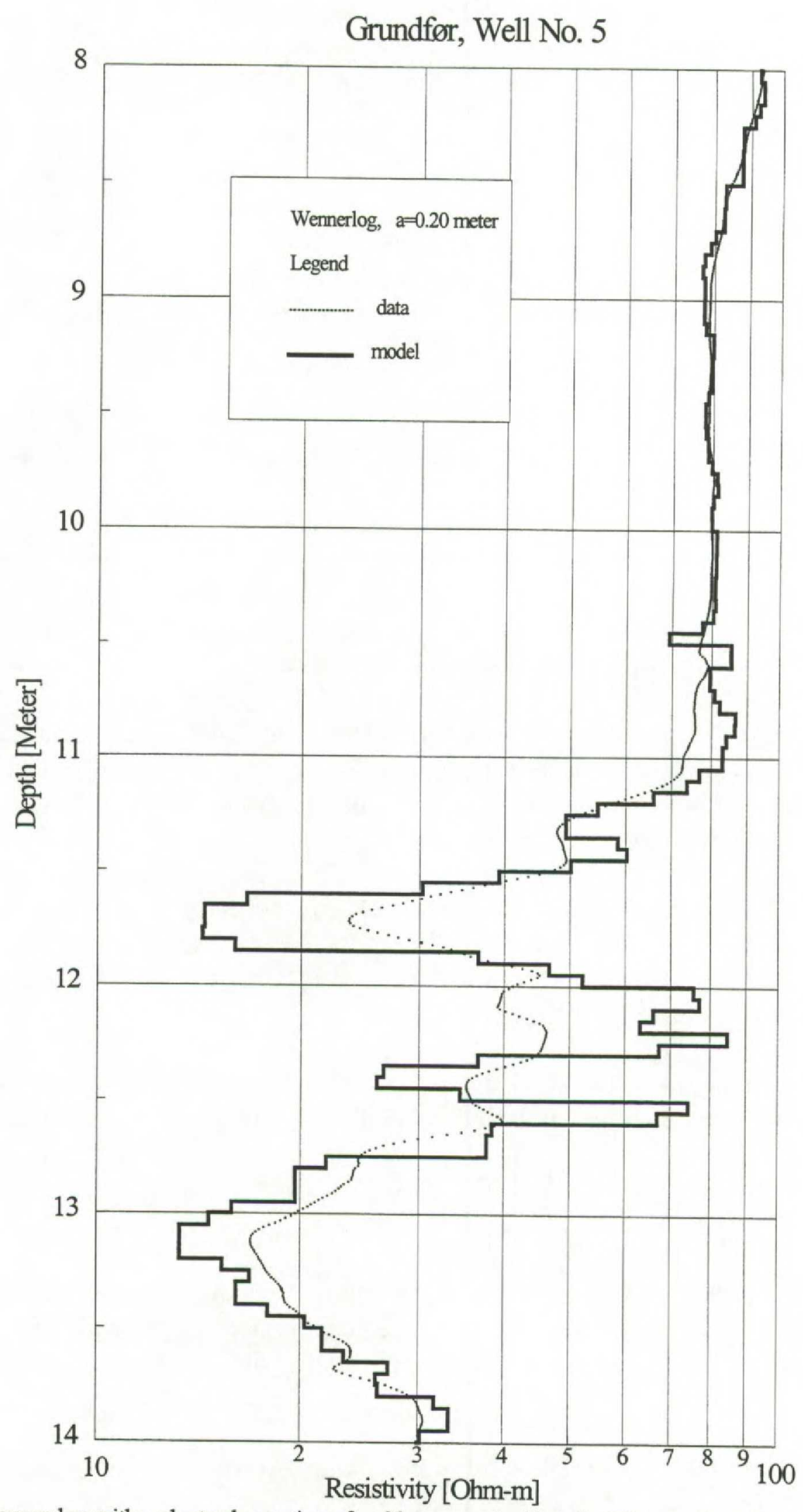

Figure 2. A Wenner $\log$ with a electrode spacing of $a=20 \mathrm{~cm}$ acquired by the Ellog method (Sørensen, 1989).

(Sørensen, 1989). The well is located close to Aarhus, Denmark, where the geology is dominated by quaternary sand, till and clay deposits. The electrode spacing is $20 \mathrm{~cm}$ and the data density is one data point per $\mathrm{cm}$. The ellog is acquired while auger drilling, which gives continuous measurements and no invasion zone. For that reason, the 1D-model is a good 
descriptive type of model.

It can be seen that in regions where the apparent resistivity varies slowly, the model result is similar to the apparent resistivity. In regions where the apparent resistivity is shifting rapidly, a significant difference can be seen.

From 11 to 13 metres the inverted log shows some thin sand and clay layers. These thin layers can be of crucial importance for the position of the natural redox boundaries in the groundwater as well as the distribution of contaminate plumes and the flow patterns achievable in pump and treat remediation.

\section{DISCUSSION AND CONCLUSION}

The resolution of electrical logs can be improved considerably by inversion of the measured apparent resistivity data. The 1-D L1-norm MLM inversion method, presented in this paper, works automatically and is independent on the initial model. Therefore, no user interaction is necessary, which leads to reliable and objective method. The improved resolution has from a chemical point of view turned out to be of vital importance.

Another point is that the inversion is very time consuming. But because the method is fully automatic, it is not a problem, as the modelling can be done e.g. at night. If a faster data processing is desired, an approximative method can be chosen. Such a method is dependent on a relative small resistivity contrasts, which very seldom is fulfiled.

The method presented in this paper was developed for the purpose of processing electrical logs acquired with the Ellog (Sørensen, 1989). But electrical logs with other kind of equipment acquired in all kind of electrode configurations can be inverted. The routine however does not compensate for the invasion zone.

In the future, a joint inversion of inductive and electrical logs will be natural. This will improve the resolution and combine the individual forces of two complimentary methods.

\section{REFERENCES}

Christensen, N.B., and Auken, E., 1992. SELMA - Simultaneous electromagnetic layered modelling and analysis. In: Jacobsen, B.H. (ed.): Proceedings of Interdisciplinary Inversion Workshop 1 Aarhus 1992, Methodology and Applications within Gephysics, Astronomy, and Geodesy. GeoSkrifter, Aarhus University, 41, p.49-56.

Madsen, K. and Nielsen, H.B., 1993. A finite smoothing algorithm for linear L1 estimation, Siam J. Optimization, Vol. 3, No. 2, p. 223-235.

Sørensen, K. I., 1989. A method for measurement of the electrical formation resistivity while auger drilling, First Break, 7, No. 10, p.403-407. 